\title{
Introduction to the Acta IMEKO special issue on the 'IMEKO TC3, TC5 and TC22 International Conference 2017'
}

\author{
Maija Ojanen-Saloranta ${ }^{1}$, Petri Koponen ${ }^{2}$ \\ ${ }^{1}$ VTT MIKES, Tekniikantie 1, Espoo, P.O.Box 1000, FI-02044 VTT, Finland \\ 2 VTT MIKES, Tehdaskatu 15, Puristamo 9P19, FI-87100 Kajaani, Finland
}

Section: Editorial

Citation: Maija Ojanen-Saloranta, Petri Koponen, Introduction to the Acta IMEKO special issue on the 'IMEKO TC3, TC5 and TC22 International Conference 2017', Acta IMEKO, vol. 8, no. 1, article 1, March 2019, identifier: IMEKO-ACTA-08 (2019)-01-01

Editor: Dušan Agrež, University of Ljubljana, Slovenia

Received March 28, 2019; In final form March 28, 2019; Published March 2019

Copyright: ( 2019 IMEKO. This is an open-access article distributed under the terms of the Creative Commons Attribution 3.0 License, which permits unrestricted use, distribution, and reproduction in any medium, provided the original author and source are credited.

Corresponding author: Petri Koponen, e-mail: petri.koponen@vtt.fi

\section{Distinguished Readers,}

The here finalized issue of Acta IMEKO presents the highlights of the Joint Conference of IMEKO Technical Committees 3 (Measurement of Force, Mass and Torque), 5 (Hardness Measurement), and 22 (Vibration Measurement). The conference was hosted by the Finnish Society of Automation, and took place on May 30 to June 1, 2017, in spring like but still chilly Helsinki. This event invited experts and presentations around the theme "Measurements facing new challenges". Out of the 97 presented papers, from 24 countries, the section chairpersons and members of the Scientific Committee made the selection of the most suitable 17 papers, with the standards, requirements and profile of Acta IMEKO Journal. As result of the review process with highly committed referees, the extended versions of the best 5 papers were accepted for this issue.

Schiavi et al. presented an experimental procedure and calculation model for the measurement of the indentation modulus by using the primary hardness standard machine at INRiM in the macro-scale range at room temperature. The indentation modulus calculation is based on the Doerner-Nix linear model and from accurate measurements of indentation load, displacement, contact stiffness, and hardness indentation imaging. The geometrical dimensions of both the Vickers and Brinell indentations are measured by means of a micromechanical system and optical microscopy imaging techniques.

A method for the evaluation of the response of torque transducers to dynamic load profiles is proposed by Oliveira et al. This paper presents a method for providing torque traceability to rotating sensors under high torque variation rates using the principle of applying acceleration pulses to rotating shafts, with mounted reference discs with known mass moments of inertia. The research gives a very good idea of the necessary different future approaches to guarantee traceability for mechanical quantities.
A set-up for the dynamic calibration of bridge amplifiers from DC up to $10 \mathrm{kHz}$ was presented by Klaus. This paper describes a dynamic bridge amplifier calibration set-up based on the new PTB dynamic bridge standard. The calibration is carried out by synchronous sampling of the bridge amplifier output voltage and a reference signal provided by the calibrated dynamic bridge standard. The dynamic bridge standard enables calibrations in a frequency range from DC (static calibration) up to $10 \mathrm{kHz}$.

A new method for the calibration of strain cylinders using laser interferometry was presented by Cui et al. Their research investigates the possibility of calibrating strain cylinders using laser interferometry, thus providing a new type of transducer that can provide both force and deformation indications. This new method for the calibration of strain cylinders is based on the application of a dual channel laser interferometer in a force standard machine. Using the proposed new method, the relationship between force, deformation, and strain can be calibrated in parallel when calibration forces are applied according to the procedure outlined in ISO 376.

Liu et al. presented a tri-axial primary vibration calibration system that has been set up at the Chinese National Institute of Metrology for simultaneous calibration of motion transducers. The system is driven by three electrodynamics exciters that are mounted along the three orthogonal axes. The tri-axial measuring system can simultaneously measure the three orthogonal vibration quantities based on the band-pass sampling method. The results show that a variety of spatial orbits can be generated by efficiently reducing the cross-coupling of the triaxial vibration exciter.

In addition to presenting new, innovative approaches to calibration work, the selected articles address the challenges and experimentation in dynamic calibrations and measurements field whose importance is becoming increasingly important and where the SI traceability must be brought. 
We are deeply honoured to be able to give our contribution to the measurement science community to act as Guest Editors for this innovative and challenging issue of Acta IMEKO. The here presented release is the result of hard but rewarding scientific work, with authors, reviewers, copy-editors, layout editors and, last but not least, the Editor-in-Chief, Prof. Dušan Agrež, providing his support for all this period.

Maija Ojanen-Saloranta

Petri Koponen

Guest Editors

\section{Editorial to additional papers}

Dear Reader,

Eight additional papers are closing the first issue of Acta IMEKO in 2019.

The paper submitted by Tarabini et al. is to deal with the possibility of monitoring the activity of and falls among people in dwellings using three or more accelerometers fixed on the ground. The main improvement of the proposed method is that it uses acceleration to estimate the impact force by using the apparent mass of the floor, experimentally identified in each room in which the tests were performed and by applying the heel drop test. The results show the possibility of identifying the presence of subjects inside the room and the falls in the majority of dwellings.

De Zanet et al. present measurement problems with accurate estimation of thrombus volume, which is an important quantity for estimation as a significant index related to the individual thrombotic risk profile. In 3D confocal microscopy, systematic errors can arise from distortions of the axial distance, whose accurate calibration remains a challenge. As a result, the $3 \mathrm{D}$ reconstructions show a noticeable axial elongation, and the volume measurements are thus overestimated. The adaptive algorithm proposed leads to the automatic compensation of the elongation error and to the accurate thrombus volume measurement.

The paper submitted by Affanni describes the design and characterisation of a dual-channel electrodermal activity (EDA) and ECG sensor for acquiring data from the hands. This choice reduces the invasiveness of ECG measurement with respect to the usual vests or chest bands. The characterisation demonstrates high-level performance of the sensor in terms of linearity and jitter, even if the measurement on the hands provides a weaker ECG signal with respect to chest derivations.

Shestakov in his paper presents an overview of the directions in the development of dynamic measurement methods. He analyses the concept of dynamic measurements and outlines a short historical background. The author describes several types of dynamic characteristics of measuring equipment, which fully characterise their dynamic properties. Additionally, he analyses the types of dynamic errors and their corrections, and in conclusion, an overview of the identification of dynamic characteristics is given.

The aim of the paper submitted by Kekalainen is to deal with a time delay estimation method based on the discrete time domain approach. In this dual-channel time delay estimation model, the criterion function compares the time differences of time sequences between channels, not the magnitude values of time functions as in the conventional cross-correlation method. An estimation task is formulated as an extreme value problem in discrete index space. Thus, there are no theoretical problems in the determination of the average time delay or velocity in the non-constant or changing time delay case as long as a sufficient statistical correlation exists between channel signals.

Spagnolo et al. focused on the ability to process the image, what is a crucial skill in many measurement activities. In image processing or pattern recognition, Fast Fourier Transform (FFT) is widely used. In applications in which the use of memory is a critical element, Fast Hartley Transform (FHT) seems to be a good substitute. Authors present a comparison of the implementations of the phase correlation function using FFT and FHT.

Shading is one of the most critical factors that produces a reduction in power in photovoltaic (PV) modules. The main aim of work presented by Bucci et al. is the development of a testing procedure for the performance evaluation of commercial PV modules in the presence of partial shading on one cell. Tests were carried out using thermographic and electric measurements and by varying the shading levels according to IEC standards. As a case study, a complete characterisation of a $180 \mathrm{Wp}$ polycrystalline PV module was performed according to the proposed testing procedure.

The paper submitted by Baranov et al. presents a new lock-in amplifier with a differential input. The lock-in amplifier has a high common-mode rejection ratio of $200 \mathrm{~dB}$ at $1 \mathrm{kHz}$ and 160 $\mathrm{dB}$ in the frequency range of $20 \mathrm{~Hz}$ to $100 \mathrm{kHz}$. Authors suggest a method for extending the dynamic range of comparing signals supported by experimental results of metrological characteristic tests.

Hope you will have an interesting and a fruitful reading of this issue of Acta IMEKO.

Dušan Agrež, Editor-in-Chief of Acta IMEKO 